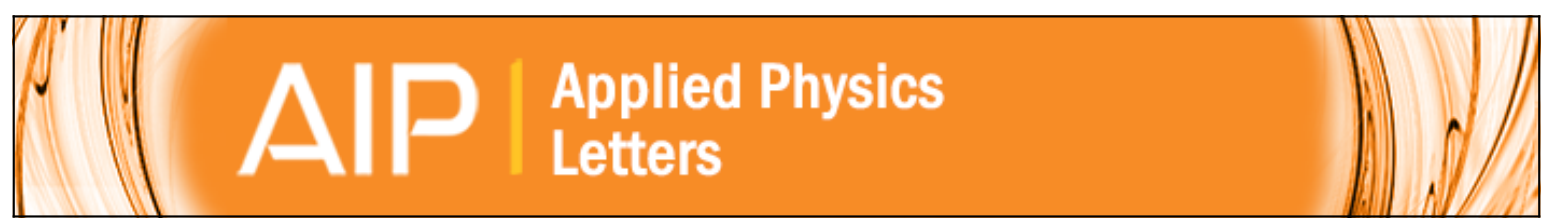

\title{
On the energy harvesting potential of piezoaeroelastic systems
}

A. Erturk, W. G. R. Vieira, C. De Marqui Jr., and D. J. Inman

Citation: Applied Physics Letters 96, 184103 (2010); doi: 10.1063/1.3427405

View online: http://dx.doi.org/10.1063/1.3427405

View Table of Contents: http://scitation.aip.org/content/aip/journal/apl/96/18?ver=pdfcov

Published by the AIP Publishing

\section{Articles you may be interested in}

Frequency up-converted wide bandwidth piezoelectric energy harvester using mechanical impact J. Appl. Phys. 114, 044902 (2013); 10.1063/1.4816249

Hybrid piezoelectric-inductive flow energy harvesting and dimensionless electroaeroelastic analysis for scaling

Appl. Phys. Lett. 102, 044101 (2013); 10.1063/1.4789433

A review on frequency tuning methods for piezoelectric energy harvesting systems

J. Renewable Sustainable Energy 4, 062703 (2012); 10.1063/1.4766892

Hydroelastic response and energy harvesting potential of flexible piezoelectric beams in viscous flow

Phys. Fluids 24, 054106 (2012); 10.1063/1.4719704

Enhancing mechanical energy harvesting with dynamics escaped from potential well Appl. Phys. Lett. 100, 114107 (2012); 10.1063/1.3694272

\section{AlP Re-register for Table of Content Alerts}




\title{
On the energy harvesting potential of piezoaeroelastic systems
}

\author{
A. Erturk, ${ }^{1, a)}$ W. G. R. Vieira, ${ }^{2}$ C. De Marqui, Jr., ${ }^{2}$ and D. J. Inman ${ }^{1}$ \\ ${ }^{1}$ Department of Mechanical Engineering, Center for Intelligent Material Systems and Structures, \\ Virginia Polytechnic Institute and State University, Blacksburg, Virginia 24061, USA \\ ${ }^{2}$ Department of Aeronautical Engineering, Engineering School of São Carlos, University of São Paulo, \\ São Carlos, 13566 São Paulo, Brazil
}

(Received 24 March 2010; accepted 16 April 2010; published online 7 May 2010)

This paper investigates the concept of piezoaeroelasticity for energy harvesting. The focus is placed on mathematical modeling and experimental validations of the problem of generating electricity at the flutter boundary of a piezoaeroelastic airfoil. An electrical power output of $10.7 \mathrm{~mW}$ is delivered to a $100 \mathrm{k} \Omega$ load at the linear flutter speed of $9.30 \mathrm{~m} / \mathrm{s}$ (which is $5.1 \%$ larger than the short-circuit flutter speed). The effect of piezoelectric power generation on the linear flutter speed is also discussed and a useful consequence of having nonlinearities in the system is addressed. (C) 2010 American Institute of Physics. [doi:10.1063/1.3427405]

Vibration-based energy harvesting using piezoelectric transduction has been investigated by several researchers over the past decade. ${ }^{1}$ Typically, cantilevers with piezoceramics are used as piezoelectric energy harvesters and the source of excitation is assumed to be base motion. ${ }^{2-6} \mathrm{Re}-$ searchers have investigated linear ${ }^{2,3}$ and nonlinear ${ }^{4-6}$ piezoelectric energy harvesting under deterministic and nondeterministic excitations.

Piezoelectric energy harvesting from aeroelastic vibrations has been studied by a few authors and limited archived work exists. The literature includes investigations of energy harvesting from the flapping of piezoelectric films ${ }^{7}$ and cantilever arrays ${ }^{8}$ located behind bluff bodies. The use of a curved airfoil section with macrofiber composite piezoceramics for energy harvesting was reported by Erturk et al. ${ }^{9}$ Later at other conferences, Bryant and Garcia discussed energy harvesting from aeroelastic vibrations using an airfoil section attached to a cantilever theoretically ${ }^{10}$ and experimentally. ${ }^{11}$ De Marqui et al. ${ }^{12}$ developed finite element models for piezoelectric energy harvesting using cantilevered plates under airflow excitation.

This paper presents an experimentally validated piezoaeroelastic model with a focus on the generated electrical power and its effect on the aeroelastic response. Lumpedparameter wing-section models are very appealing due to their physical simplicity and the fundamental insight they provide. ${ }^{13}$ Therefore, the model and the experiments are given for a modified typical section undergoing selfsustained oscillations at the neutral stability condition. While the analysis given here is linear, a useful consequence of having nonlinearities in the piezoaeroelastic system is also discussed.

Consider the piezoaeroelastic section under airflow excitation shown in Fig. 1. After introducing piezoelectric coupling to the plunge degree of freedom (DOF) in addition to two structural damping coefficients and considering a resistive load in the electrical domain, the lumped-parameter aeroelastic equations ${ }^{13}$ are modified to obtain the following piezoaeroelastic equations:

$$
\left(m+m_{f}\right) \ddot{h}+m x_{\alpha} b \ddot{\alpha}+d_{h} \dot{h}+k_{h} h-\theta v / \ell=-L,
$$

\footnotetext{
a) Author to whom correspondence should be addressed. Electronic mail: erturk@vt.edu.
}

$$
\begin{aligned}
& m x_{\alpha} b \ddot{h}+I_{p} \ddot{\alpha}+d_{\alpha} \dot{\alpha}+k_{\alpha} \alpha=M, \\
& C_{p}^{\mathrm{eq}} \dot{v}+v / R_{l}+\theta \dot{h}=0,
\end{aligned}
$$

where $h$ is the plunge displacement (translation), $\alpha$ is the pitch displacement (rotation), $m$ is the airfoil mass per length (in the span direction), $m_{f}$ accounts for the fixture mass per length in the experiments connecting the airfoil to the plunge springs $\left(m_{f}=0\right.$ for the ideal representation given in Fig. 1$), I_{p}$ is the moment of inertia per length about the reference point $P$ where $h$ is measured, $b$ is the semichord length, $\ell$ is the span length (into the page), $x_{\alpha}$ is the dimensionless chordwise offset of the reference point from the centroid (point $C$ ), $k_{h}$ is the stiffness per length in the plunge DOF, $k_{\alpha}$ is the stiffness per length in the pitch DOF, $L$ is the aerodynamic lift per length, $M$ is the aerodynamic pitching moment per length, $d_{h}$ and $d_{\alpha}$, respectively, are the structural damping coefficients in the plunge DOF and the pitch DOF, $R_{l}$ is the load resistance, $v$ is the voltage across the resistive load, $C_{p}^{\mathrm{eq}}$ is the equivalent capacitance of the piezoceramic layers, and $\theta$ is the electromechanical coupling term and an over-dot represents differentiation with respect to time.

Assuming harmonic response at frequency $\omega$ (i.e., $h=\bar{h} e^{j \omega t}, \alpha=\bar{\alpha} e^{j \omega t}, v=\bar{v} e^{j \omega t}, L=\bar{L} e^{j \omega t}$, and $M=\bar{M} e^{j \omega t}$ where $j=\sqrt{-1}$ ) leads to the following complex eigenvalue problem for the steady-state plunge and pitch displacements:

$$
\left[\beta+\frac{\ell_{h}}{\mu}-\kappa(\omega)-\sigma^{2}\left(1+j \gamma_{h}\right) \lambda\right] \frac{\bar{h}}{b}+\left(x_{\alpha}+\frac{\ell_{\alpha}}{\mu}\right) \bar{\alpha}=0,
$$

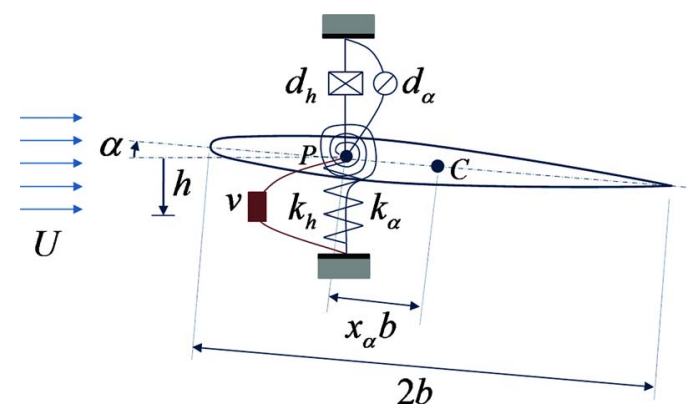

FIG. 1. (Color online) Schematic of a piezoaeroelastic section under uniform airflow. 


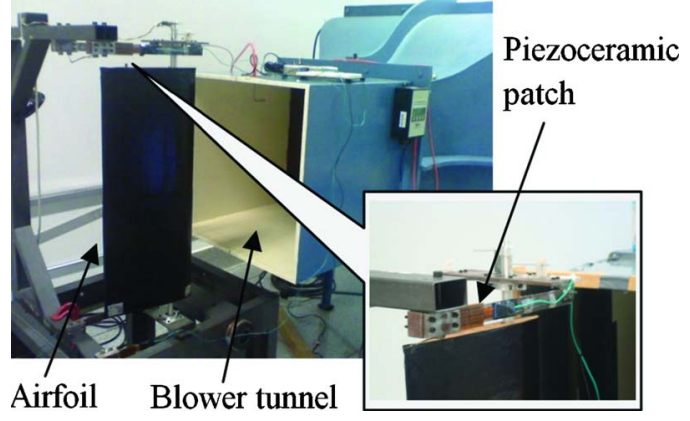

FIG. 2. (Color online) Experimental setup showing a typical aeroelastic section with piezoceramics attached onto the plunge stiffness members.

$$
\left(x_{\alpha}+\frac{m_{h}}{\mu}\right) \frac{\bar{h}}{b}+\left[r^{2}+\frac{m_{\alpha}}{\mu}-r^{2}\left(1+j \gamma_{\alpha}\right) \lambda\right] \bar{\alpha}=0,
$$

where the aerodynamic loads $\left(\ell_{h}, \ell_{\alpha}, m_{h}\right.$, and $\left.m_{\alpha}\right)$ are taken from Theodorsen's unsteady thin airfoil theory ${ }^{14}$ and are functions of the reduced frequency $(k=\omega b / U$ where $U$ is the airflow speed) and the geometric parameters. It is important to note that, in this linear model, the harmonic response assumption holds for the condition of neutral stability only [i.e., Eqs. (4) and (5) are valid at the classical flutter boundary only]. The dimensionless terms are the complex eigenvalue, $\lambda=\left(\omega_{\alpha} / \omega\right)^{2}$, the frequency ratio, $\sigma=\omega_{h} / \omega_{\alpha}$ (where $\omega_{h}=\sqrt{k_{h} / m}$ and $\omega_{\alpha}=\sqrt{k_{\alpha} / I_{p}}$ ), the dimensionless radius of gyration, $r=\sqrt{I_{p} / m b^{2}}$, the airfoil-to-affected air mass ratio, $\mu=m / \pi \rho b^{2}$ (where $\rho$ is the free-stream air mass density), and a mass ratio that accounts for the presence of a fixture between the airfoil and the plunge springs, $\beta=(m$ $\left.+m_{f}\right) / m$. The loss factors in Eqs. (4) and (5) are assumed to obey $\gamma_{h}=\omega d_{h} / k_{h}$ and $\gamma_{\alpha}=\omega d_{\alpha} / k_{\alpha}$, and they are identified at zero airflow speed.

The dimensionless term $\kappa(\omega)$ in Eq. (4) is due to eliminating the voltage term using Eq. (3) in Eq. (1) and it depends on the eigenvalue $\lambda$ since it is a function of frequency: $\kappa(\omega)=j \theta^{2}\left(j \omega C_{p}^{\mathrm{eq}}+1 / R_{l}\right)^{-1} /(\omega m \ell)$. Hence an iterative solution procedure is required where the frequency to be used in $\kappa(\omega)$ is obtained from the eigenvalue that becomes unstable with increasing airflow speed. The convergence of the iterative eigensolution is extremely fast if one starts with the solution of the piezoelectrically uncoupled aeroelastic problem $[\kappa(\omega)=0]$. Once the complex eigenvector relationship between $\bar{h}$ and $\bar{\alpha}$ is obtained, $\bar{v}$ is calculated using

$$
\bar{v}=-j \omega \theta\left(j \omega C_{p}^{\mathrm{eq}}+1 / R_{l}\right)^{-1} \bar{h} .
$$

For a given load resistance, the airflow speed that makes the imaginary part of the respective eigenvalue branch zero is the linear flutter speed $\left(U=U_{c}\right)$ and the piezoaeroelastic ei-

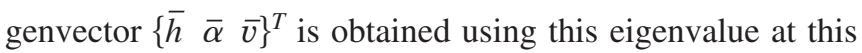
particular speed.

Figure 2 shows the experimental setup used for investigating the piezoaeroelastic response of an airfoil section. The system parameters are $x_{\alpha}=0.260, r=0.504$, $\beta=2.597, \sigma=3.33, \mu=29.6, \quad b=0.125 \mathrm{~m}, \ell=0.5 \mathrm{~m}$, and $\omega_{\alpha}=15.4 \mathrm{rad} / \mathrm{s}$. The loss factors identified for the plunge DOF and the pitch DOF at zero airflow speed are $\gamma_{h}$ $=0.007$ and $\gamma_{\alpha}=0.12$. A dimensionless geometric parameter required for the Theodorsen function ${ }^{13,14}$ is the relative location of the reference point with respect to the midchord and

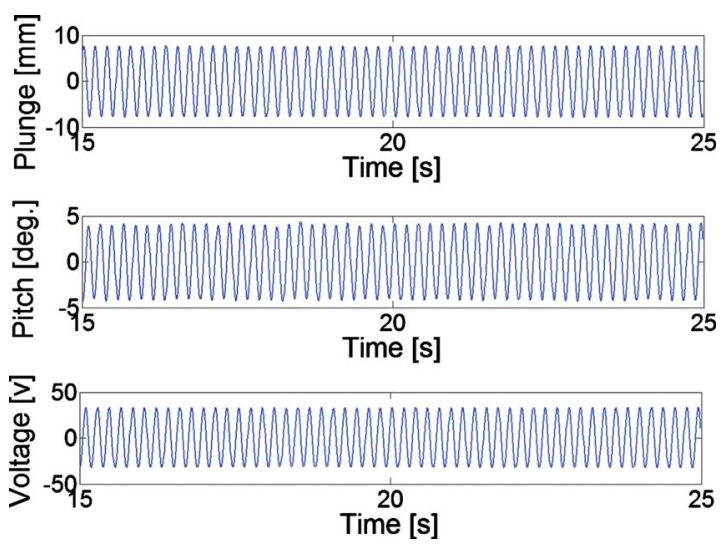

FIG. 3. (Color online) Experimental piezoaeroelastic response for $R_{l}=100 \mathrm{k} \Omega$ and $U_{c}=9.30 \mathrm{~m} / \mathrm{s}$.

it is $a=-0.5$ for this setup. The plunge stiffness of the airfoil is due to four steel beams (in clamped-clamped end conditions) connecting the airfoil to the ground from the reference point. Two PZT-5A piezoceramics (QP10N from Midé Technology Corporation) are attached onto the roots of two of these beams symmetrically and their electrodes are combined in parallel. The electromechanical coupling term is obtained based on distributed-parameter modeling ${ }^{2}$ as $\theta=1.55 \mathrm{mN} / \mathrm{V}$ and the manufacturer's published equivalent capacitance of $C_{p}^{\mathrm{eq}}=120 \mathrm{nF}$ is used in the model. In the experiments, the airflow speed is slowly increased from zero until almost persistent piezoaeroelastic response is obtained for each resistive load.

The short-circuit $\left(R_{l} \rightarrow 0\right)$ and the open-circuit $\left(R_{l} \rightarrow \infty\right)$ flutter speeds are measured as $U_{c}^{\mathrm{sc}}=8.85 \mathrm{~m} / \mathrm{s}$ and $U_{c}^{\mathrm{oc}}$ $=8.90 \mathrm{~m} / \mathrm{s}$, respectively. Figure 3 shows the piezoaeroelastic response for an electrical load resistance of $100 \mathrm{k} \Omega$ with almost persistent oscillations approximately at the linear flutter speed of $9.30 \mathrm{~m} / \mathrm{s}$. Among the set of resistors used in the experiments, this is the electrical load that gives the maximum power output $(10.7 \mathrm{~mW})$. For this electrical load, the absolute value of the normalized piezoaeroelastic eigenvector is obtained from the model as $\{|\bar{h}||\bar{\alpha}||\bar{v}|\}^{T}$ $=\left\{1 \mathrm{~mm} 0.56^{\circ} / \mathrm{mm} 4.67 \mathrm{~V} / \mathrm{mm}\right\}^{T}$ at the flutter speed of $9.56 \mathrm{~m} / \mathrm{s}$. The experimental maximum response amplitudes in Fig. 3 are $|\bar{h}|=7.65 \mathrm{~mm},|\bar{\alpha}|=4.18^{\circ}$, and $|\bar{v}|=32.7 \mathrm{~V}$. Hence the experimental ratios $|\bar{\alpha}| /|\bar{h}|=4.18 / 7.65$ $=0.55^{\circ} / \mathrm{mm}$ and $|\bar{v}| /|\bar{h}|=32.7 / 7.65=4.27 \mathrm{~V} / \mathrm{mm}$ exhibit good agreement with the model.

The linear flutter speed for $R_{l}=100 \Omega$ (close to shortcircuit conditions) is predicted by the model as $9.06 \mathrm{~m} / \mathrm{s}$, overestimating the experimental value of $8.85 \mathrm{~m} / \mathrm{s}$ by $2.4 \%$. The model predicts the linear flutter speed for $R_{l}=100 \mathrm{k} \Omega$ as $9.56 \mathrm{~m} / \mathrm{s}$, which overestimates the experimental value of 9.30 $\mathrm{m} / \mathrm{s}$ by $2.8 \%$. The flutter frequency is obtained by the model as $5.17 \mathrm{~Hz}$ for $R_{l}=100 \Omega$ (underestimating the experimental value by $1.7 \%$ ) and as $5.14 \mathrm{~Hz}$ for $R_{l}=100 \mathrm{k} \Omega$ (underestimating the experimental value by $2.3 \%$ ).

Figure 4(a) shows the voltage-to-plunge displacement amplitude ratio while Fig. 4(b) shows the pitch-to-plunge displacement amplitude ratio for the set of resistors used in the experiment along with the theoretical predictions. The voltage-to-plunge displacement versus load resistance curve exhibits linear asymptotes similar to the trend in the harmonic base excitation of piezoelectric energy harvesters ${ }^{2}$ 
(a)

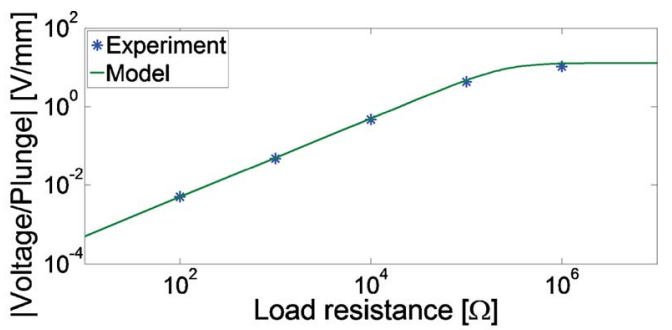

(b)

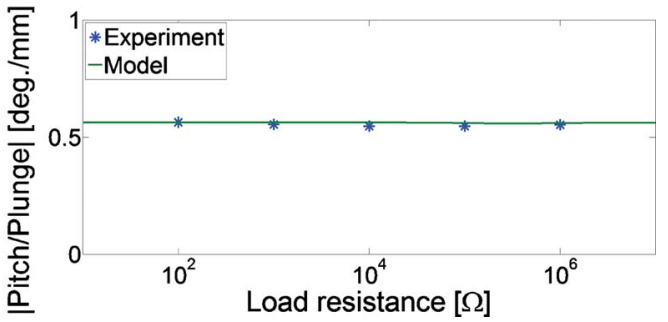

FIG. 4. (Color online) Theoretical and experimental (a) voltage output-toplunge displacement and (b) pitch displacement-to-plunge displacement ratios vs load resistance.

whereas the variation in the pitch-to-plunge displacement amplitude is remarkably insensitive to the changing load resistance. It should be highlighted that these theoretical and experimental data points are given for the flutter speed that corresponds to the respective load resistance (e.g., for $R_{l}=10 \mathrm{k} \Omega, \quad|\bar{h}|=5.15 \mathrm{~mm},|\bar{\alpha}|=2.82^{\circ}$, and $|\bar{v}|=2.42 \mathrm{~V}$ whereas for $R_{l}=1 \mathrm{M} \Omega,|\bar{h}|=7.95 \mathrm{~mm},|\bar{\alpha}|=4.40^{\circ}$, and $|\bar{v}|$ $=83.1 \mathrm{~V})$.

The electrical power-to-plunge displacement ratio versus load resistance curve is shown in Fig. 5(a). The optimal load that gives the maximum power output causes a considerable increase in the linear flutter speed [Fig. 5(b)] due to the shunt damping effect ${ }^{15}$ of piezoelectric power generation. The experimental increase in the linear flutter speed (with respect to the short-circuit flutter speed) for $R_{l}=100 \mathrm{k} \Omega$ is $5.1 \%$ and the model predicts this increase as 5.5\% in Fig. 5(b). Therefore, piezoelectric energy harvesting has the favorable effect of increasing the flutter speed of the piezoaeroelastic system.

Often nonlinearities are present in aeroelastic systems in
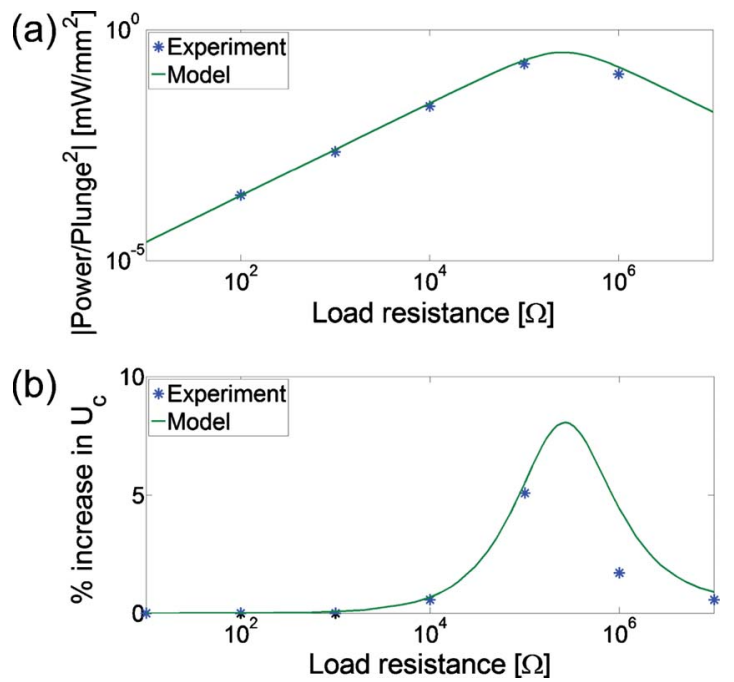

FIG. 5. (Color online) Theoretical and experimental (a) normalized power and (b) percentage increase in the flutter speed vs load resistance. (a)

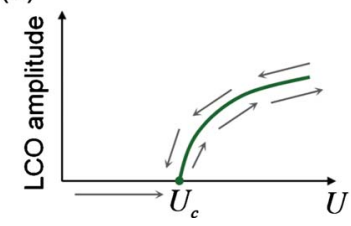

(b)

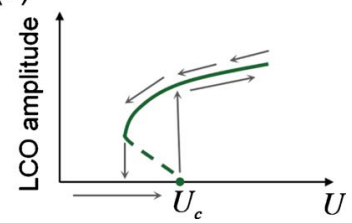

FIG. 6. (Color online) Two types of LCO response due to (a) supercritical and (b) subcritical bifurcations with changing airflow speed $\left(U=U_{c}\right.$ is the linear flutter speed).

the forms of (1) free play or bilinear stiffness due to loosely connected components, (2) material and geometric nonlinearity (typically yielding nonlinear stiffness effect), and (3) dry friction and other forms of nonlinear damping. ${ }^{16}$ The presence of such nonlinearities may result in limit-cycle oscillations (LCO) at airflow speeds above [Figs. 6(a) and 6(b)] or below [Fig. 6(b)] the linear flutter speed. The LCO mechanism in Fig. 6(b) leads to both stable (solid line) and unstable (dashed line) LCO below the linear flutter speed and it is not preferred in real aircraft. ${ }^{16}$ However, this type of LCO might be useful in a piezoaeroelastic system designed for energy harvesting only ${ }^{10,11}$ so that large-amplitude response can be obtained for a wider range of airflow speeds. A typical section similar to the one in Fig. 1 with a nonlinear stiffness component and/or a free play can be used to investigate LCO for piezoelectric energy harvesting and theoretical tools are available to solve the resulting nonlinear equations for the limit cycles. ${ }^{17}$ Although the focus in this paper has been placed on linear flutter, stable LCO of acceptable amplitude in nonlinear piezoaeroelastic systems can provide an important source of persistent electrical power and it deserves separate investigation.

The authors gratefully acknowledge the support from the AFOSR under Grant Nos. F 9550-06-1-0326 and F 9550-091-0625 monitored by Dr. B. L. Lee, and the support from CNPq and FAPEMIG through INCT-EIE.

${ }^{1}$ K. A. Cook-Chennault, N. Thambi, and A. M. Sastry, Smart Mater. Struct. 17, 043001 (2008).

${ }^{2}$ A. Erturk and D. J. Inman, Smart Mater. Struct. 18, 025009 (2009).

${ }^{3}$ S. Adhikari, M. I. Friswell, and D. J. Inman, Smart Mater. Struct. 18, 115005 (2009).

${ }^{4}$ L. Gammaitoni, I. Neri, and H. Vocca, Appl. Phys. Lett. 94, 164102 (2009).

${ }^{5}$ A. Erturk, J. Hoffmann, and D. J. Inman, Appl. Phys. Lett. 94, 254102 (2009).

${ }^{6}$ S. C. Stanton, C. C. McGehee, and B. P. Mann, Appl. Phys. Lett. 95, 174103 (2009).

${ }^{7}$ W. P. Robbins, D. Morris, I. Marusic, and T. O. Novak, Proceedings of the ASME IMECE 2006, Chicago, IL, 2006.

${ }^{8}$ S. Pobering, S. Ebermeyer, and N. Schwesinger, Proc. SPIE 7288, 728807 (2009).

${ }^{9}$ A. Erturk, O. Bilgen, M. Fontenille, and D. J. Inman, Proceedings of the ICAST 2008, Ascona, Switzerland, 2008.

${ }^{10}$ M. Bryant and E. Garcia, Proc. SPIE 7288, 728812 (2009).

${ }^{11}$ M. Bryant and E. Garcia, Proc. SPIE 7493, 74931W (2009).

${ }^{12} \mathrm{C}$. De Marqui, Jr., A. Erturk, and D. J. Inman, Proceedings of the ICCM 2009, Edinburgh, UK, 2009.

${ }^{13}$ D. H. Hodges and G. A. Pierce, Introduction to Structural Dynamics and Aeroelasticity (Cambridge University Press, New York, 2002).

${ }^{14} \mathrm{~T}$. Theodorsen, Langley Memorial Aeronautical Laboratory Report No. NACA-TR-496, 1935 (unpublished).

${ }^{15}$ A. Agneni, F. Mastroddi, and G. M. Polli, Comput. Struc. 81, 91 (2003).

${ }^{16}$ E. H. Dowell and D. Tang, AIAA J. 40, 1697 (2002).

${ }^{17}$ Y. M. Chen and J. K. Liu, J. Vib. Control 16, 163 (2010). 